\title{
Polarimetric Characterization and Temporal Stability Analysis of Urban Target Scattering
}

\author{
Gerard Margarit, Member, IEEE, Jordi J. Mallorquí, Member, IEEE, and Luca Pipia, Member, IEEE
}

\begin{abstract}
This paper studies the polarimetric-dispersion properties of urban targets and their evolution along time in terms of the geometrical configuration. The relations between target geometry and the scattering behavior have been defined through the analysis of large stacks of simulated images. Scattering maps and synthetic aperture radar (SAR) images have been synthesized with the numerical tool GRaphical Electromagnetic COmputing SAR for different qualitative models of two real buildings. Ground-based SAR (GB-SAR) data acquired in a subsidence measurement campaign has been used to assess the simulator's realism. These data have permitted the identification of the critical simulation parameters and their range of recommended values for realistic simulations. In the context of very high resolution images, the results derived from this study may be crucial for making progress in urban-image postprocessing. As the different resolution cells comprise few scattering centers showing a quasideterministic scattering behavior, nonprobabilistic models based on target's geometry seem more suited for scattering modeling. In these models, the geometry-scattering (GS) links precisely inferred from simulated images can be very important. In addition to change detection and land classification, GS models may help in improving the interpretation of subsidence results with differential interferometry. Certainly, new processing algorithms can be developed exploiting the available scattering data with more physical sense. In addition, they can take more advantage of the fine resolution and polarimetric capabilities of the new sensors, like TerraSAR-X or RADARSAT-2.
\end{abstract}

Index Terms-Polarimetry, synthetic aperture radar (SAR) simulation, urban scattering.

\section{INTRODUCTION}

$\mathbf{S}$ YNTHETIC aperture radar (SAR) sensing of urban areas is becoming an important research line. The improved performance of new sensors with finer resolutions and additional channels (polarimetric and/or interferometric) is increasing the sensitivity to more complex geometries. Now, small-scale details and multiple reflection can be analyzed as never before. This gives more physical sense to urban-scattering analysis.

Manuscript received February 15, 2009; revised July 28, 2009 and September 8, 2009. First published November 24, 2009; current version published March 24, 2010. This work was supported in part by the Spanish Ministry of Education and in part by the European Union FEDER funds under Project TEC2008-06764-C02-01.

G. Margarit is with the Science and Earth Observation Processing Systems, GMV Aerospace and Defense, 08006 Barcelona, Spain (e-mail: gmargarit@ gmv.com).

J. J. Mallorqui is with the Remote Sensing Laboratory, Universitat Politècnica de Catalunya (UPC), 08034 Barcelona, Spain (e-mail: mallorqui@tsc. upc.edu).

L. Pipia was with UPC, 08034 Barcelona, Spain. He is now with the Institut Cartogràfic de Catalunya, 08038 Barcelona, Spain (e-mail: lpipia@icc.cat).

Color versions of one or more of the figures in this paper are available online at http://ieeexplore.ieee.org.

Digital Object Identifier 10.1109/TGRS.2009.2035052
In this new framework, the probabilistic approaches traditionally adopted with medium-resolution SAR images seem to be not suitable for current very high resolution (VHR) data. The reason falls on the few scattering centers present within each resolution cell, which makes the scattering pattern follow a more deterministic trend. In this context, the geometrical features of the targets play an important role, and, hence, their connection with particular scattering mechanisms is essential for proper data analysis. Benefits of accurate geometry-scattering (GS) links in VHR would allow the improvement of parameter inversion, building description, height-profile retrieval [1], or guide-scatterer characterization [2], [3]. Right now, few works have tackled this issue.

In [2], stacks of Polarimetric Interferometric SAR (InSAR) (POLInSAR) images are used to characterize certain structures with coherent scatterer descriptors. Despite the interesting conclusions, the descriptor values are not supported by a geometrical and physical analyses of targets. Something similar happens in [3] where an application of urban scattering for building discrimination is presented. There, the set of optimum POLInSAR coherences [4] is used to differentiate buildings via entropy and anisotropy. Building height is then retrieved by assuming ground scatterers behaving as dihedrals whereas those on roofs as trihedrals. However, such assumptions were only based in simple observations without the support of a scattering analysis of buildings. The same applies for [5] where the proposed POLInSAR cluster classifier has a limited interpretation of the available classes.

First efforts focused to evaluate the relations between target geometry and scattering are available in [6]. The analysis of large series InSAR images with diversified acquisition geometries was used to define an urban model. The main goal was to improve the retrieval of some parameters, such as 3-D location and deformation velocities. Therefore, urban characterization was not focused to describe the scattering in terms of geometrical configuration but to improve the performance of classical Differential Interferometric SAR (DInSAR) techniques [7].

In this context, this paper makes a step forward and studies urban scattering from a geometrical point of view in a VHR context. The main goal is to characterize the polarimetric properties of certain geometries and outline their dependence with respect to some key parameters, such as orientation, incidence angle, operating frequency, and time dimension. The links between dispersion and geometrical shapes will help in improving the quantitative inversion of some urban-related parameters, such as building height or deformation. In addition, they can support a better characterization of the scattering patterns of urban targets. 


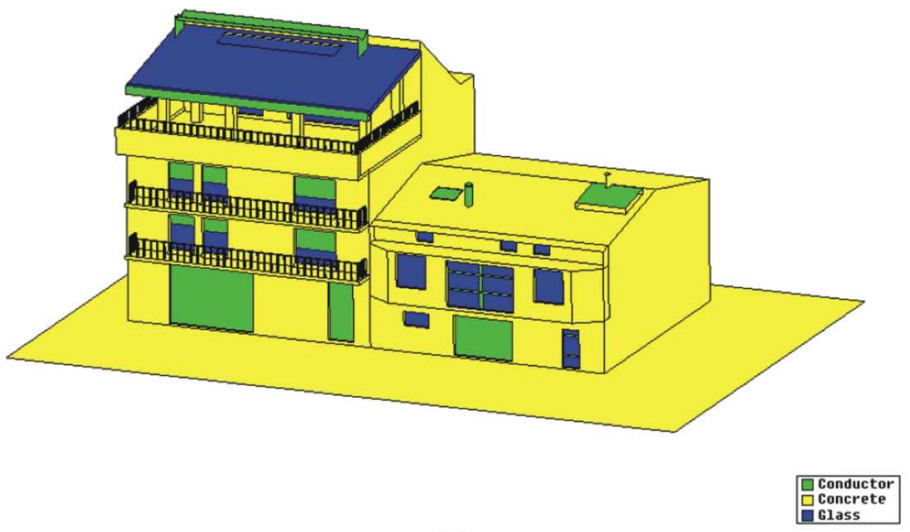

(a)

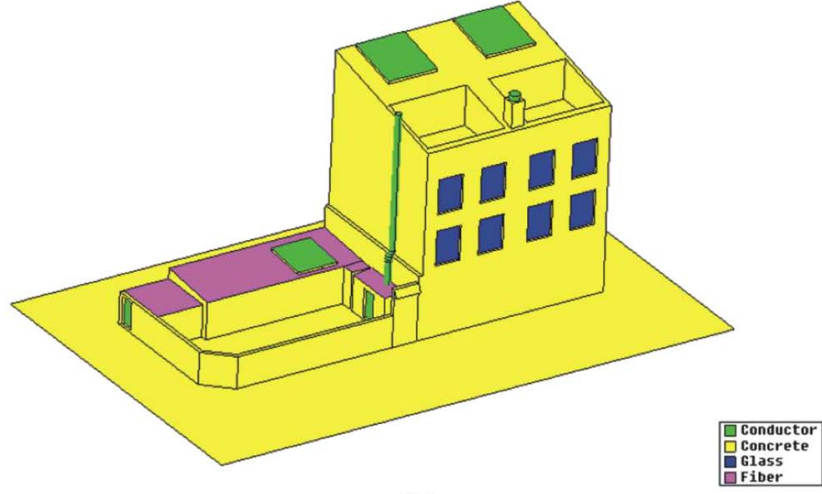

(b)

Fig. 1. Models 1 and 2 related with the buildings identified in the Sallent test site according to Fig. 2. The color code shows the adopted materials.

This paper is focused to cover the following main topics, namely: 1) the description of the simulation tool and which steps are mandatory to achieve an accurate and realistic simulation; 2) the details about GS links and how they can be inferred from simulated images; and 3) the definition of the quantitative parameterization that can be applied to GS links in order to make their application in real data easier. Note that the quantitative analysis of the improvements that GS links may provide in particular applications are beyond the scope of this paper. This is something reserved for future works where an in-depth analysis with real data has to be developed.

This paper is organized as follows. Section II presents the simulation tool GRaphical Electromagnetic COmputing SAR (GRECOSAR) and briefly outlines its main features. Details about GRECOSAR processing scheme and how realistic scenarios can be achieved are treated in Section III. Sections IV and $\mathrm{V}$ present the analysis of simulated scattering maps and SAR images for different urban models. There, first GS links are defined and initially parameterized. Section VI provides the validation of simulated data with real GB-SAR images [8], [9] and outlines which benefits can provide simulated-based GS links to VHR data interpretation.

\section{Simulation EnVIRONMENT: GRECOSAR}

GRECOSAR is a numerical tool able to synthesize the SAR signatures of complex targets with notable realism (see [10], [11] for further details). It can deal with polarimetric SAR, POLInSAR and polarimetric inverse SAR (ISAR) geometries for any operating band, mode, and system resolution in a configurable scenario. The kernel of the simulator is the Universitat Politècnica de Catalunya (UPC's) GRECO solver [12] that estimates, for each frequency, the radar cross section (RCS) of 3-D targets via high-frequency methods. Any type of complex target can be processed if modeled with parametric surfaces. ${ }^{1}$

\section{A. EM Kernel: GRECO}

ElectroMagnetic (EM) calculations are performed via a graphic-based approach for which a bitmap resident in the

\footnotetext{
${ }^{1}$ The CAD package GiD of the International Center of Numerical Methods for Engineering [13] has been adopted here.
}

RAM is generated from the input model. By using a particular illumination point of view fixed by the user-defined line-ofsight (LOS) direction, GRECOSAR renders the model with the PC graphic card and isolates the visible entities from the back-facing ones. Over these entities, EM methods are applied making RCS prediction faster and independent of the input geometry. The main EM methods used by GRECOSAR are the following.

1) Physical optics (PO) for perfectly conducting surfaces.

2) Method of equivalent currents with Ufimtsev's Physical Theory of Diffraction coefficients or Mitzner's incremental length diffraction coefficients for perfectly conducting edges.

3) Multiple-reflection analysis by a geometrical optics (GO) + PO ray-tracing algorithm. Bistatic GO is used for all reflections except the last one, for which PO is used. In curved surfaces, GO divergence factors are approximate.

These methods allow the analysis of targets of electrical size as large as $2^{n} \lambda / 16$, with a maximum phase error of $\lambda / 8$, where $n$ is the number of bits in which the distance to the observer is discretized. For a proper EM performance, input models have to be tessellated with a meshing procedure that discretizes parametric surfaces into small planar facets. This product is the input to the simulator, which is used by the PC's graphic card to generate the GRECO bitmap. In this way, two main parameters emerge to control the accuracy of EM simulation (and the demanded PC resources): the length of each facet defining the input model and the pixel size of GRECO bitmap. Both play a key role as explained later.

Several works have proved that GRECOSAR provides an accurate framework for evaluating the RCS and geometry of complex targets. This results on realistic SAR images for most of the operating modes [10], [11], [14]. The realism of such images have allowed significant progress in different research fields, for instance, ship classification where a new Vessel Classification Algorithm has been proposed [15], [16].

\section{B. Target Models and Imaging Geometry}

Two building models have been used in this work (see Fig. 1). They are qualitative versions of the two urban structures identified in the photograph of Fig. 2, which shows an area of the 


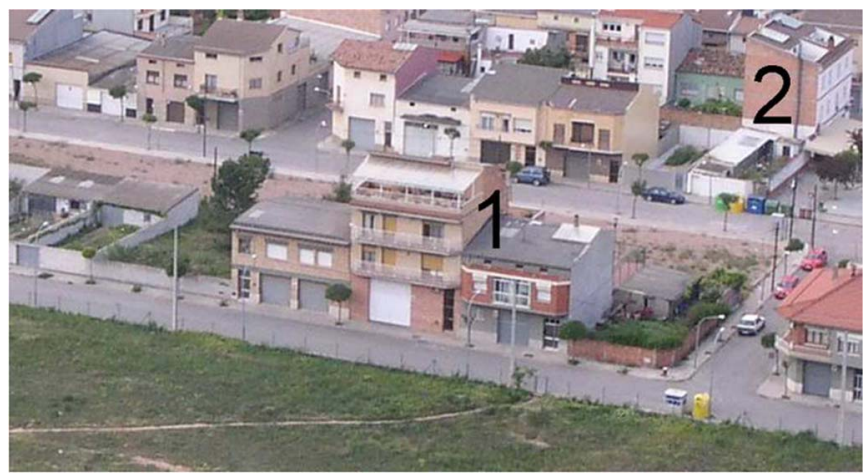

Fig. 2. Photograph of the neighborhood test site located in Sallent (50 km north from Barcelona, Spain) where subsidence monitoring with UPC's GB-SAR took place. Black numbers signal the structures modeled in Fig. 1.

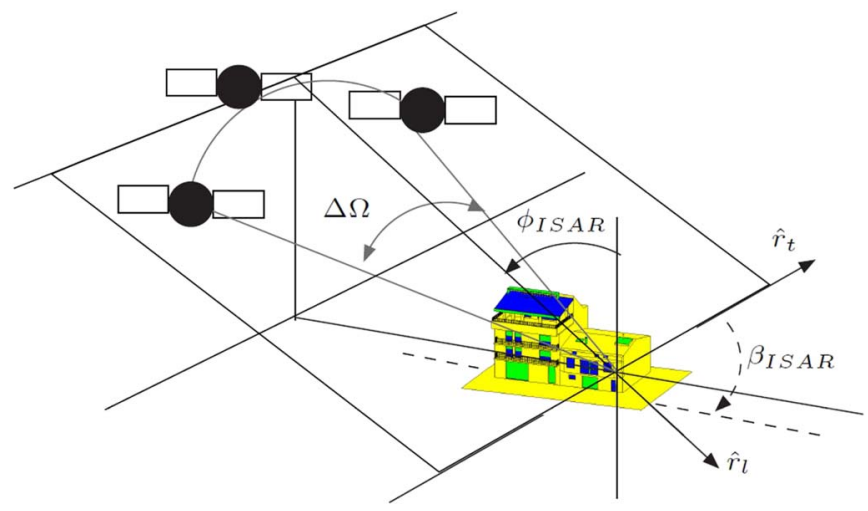

Fig. 3. ISAR imaging geometry of GRECOSAR. Sensors are in circular motion over the incidence plane $\phi_{\text {ISAR }}$, defined by the longitudinal (LOS) $\left(\hat{r}_{l}\right)$ and transversal (perpendicular) $\left(\hat{r}_{t}\right)$ direction.

GB-SAR test site [8]. ${ }^{2}$ Model dimensions and details have been deduced from the photographs with no support of blueprints.

Most of the presented images have been obtained with the ISAR imaging geometry shown in Fig. 3. It is based on the circular spotlight mode [17] where the sensor is flying in circular motion around a static target for a constant incidence angle $\phi_{\mathrm{ISAR}}$, target orientation $\beta_{\mathrm{ISAR}}$ (relative to the crossrange direction over the ground $\hat{r}_{t}$ ), and angular aperture $\Delta \Omega$. The resulting images are projected over the incidence plane as observed by a viewer located at the perpendicular axis [17]. With the suitable values of $\Delta \Omega$ and signal bandwidth, image resolutions can be fine-tuned up to the centimetric order. This gives an interesting chance for analyzing the scattering behavior of complex targets with a degree of detail almost impossible to obtain with real data.

Supporting ISAR images, simulated SAR images have been also generated according to the imaging geometry of Fig. 4. There, SAR images can be linked with the ISAR ones if $\hat{r}_{l} \| \hat{r}_{s r}$. Therefore, data cross-check becomes available.

\section{Realism Evaluation of Simulated Scenarios}

This section details the modeling and processing requirements to obtain realistic simulated images.

\footnotetext{
${ }^{2}$ It is located in Sallent, a city $50 \mathrm{~km}$ north from Barcelona (Spain), with important mining-induced subsidence.
}

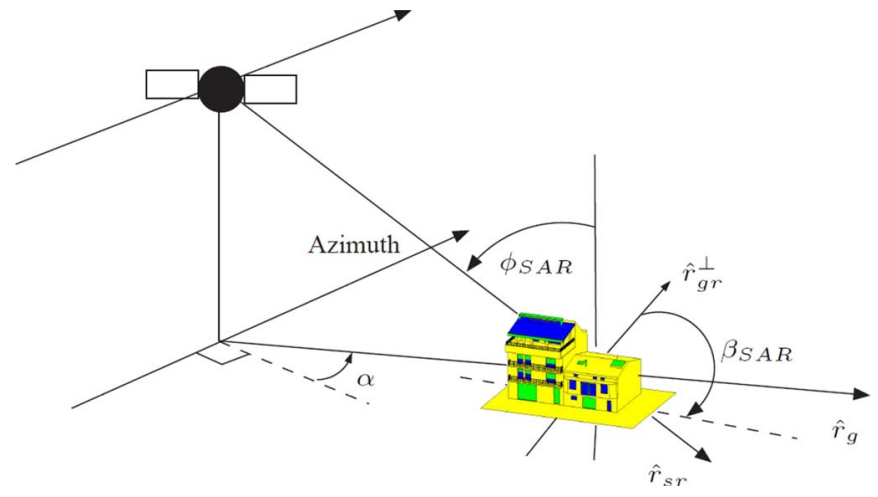

Fig. 4. SAR imaging geometry of GRECOSAR. An ideal linear track is assumed for a squint $\alpha$ and incidence $\phi_{\mathrm{SAR}}$ angle. For $\alpha=0$ and $\phi_{\mathrm{SAR}}=$ $\phi_{\mathrm{ISAR}}, \hat{r}_{s r} \| \hat{r}_{l}$ and $\hat{r}_{g r}^{\perp} \| \hat{r}_{t}$.

\section{A. Degree of Detail}

The degree of detail is probably the most critical issue. It should consider all the small-scale geometries possible without increasing in excess the computational load. If not, the resulting model will be a bad approximation of the reality similar to a boxlike shape with few scatterers. In fact, a set of experiments carried out with GRECOSAR and a perfectly flat box of gypsum located over a conductor have shown that extremely simple models generate unrealistic scattering responses [18], [19]. Few scattering centers are present, which normally behave as dihedrals. ${ }^{3}$

The influence of target details is crucial and sometimes the models have to be sophisticated even if a simple eye inspection would assume enough realism. This can be clearly observed in the ISAR scattering maps shown in Fig. 5. There, two versions of building 1 model are processed with different level of details $\left(\phi_{\mathrm{ISAR}}=60^{\circ}, \beta_{\mathrm{ISAR}}=190^{\circ}\right.$, and $f=$ $5.3 \mathrm{GHz}$ ). Their analysis with the Pauli theorem results on an RGB image where red/green/blue represents the weight of the first/second/third Pauli channel associated with (trihedral, sphere, flat plane)/dihedral/antisymmetric mechanisms. As observed, the finer the details, the higher the number of scattering centers with different behaviors. Specifically, the refined model provides an increased number of trihedral-like behaviors at the frame points of windows and doors as well as numerous small dihedral-like dots due to the posts of the banister added at the roof. Such elements give notion about the level of accuracy reached by GRECOSAR.

In this work, the degree of detail for both building models has been fixed by comparing the responses obtained for different versions with increased refinement. When the differences appear to be insignificant, no more small-scale geometries are added. The snapshots of Fig. 1 show the versions with the highest degree of detail.

\section{B. Roughness and Perfect Geometries}

Another topic of great importance lies on avoiding perfect canonical scatterers. Such geometries are unrealistic and can

\footnotetext{
${ }^{3}$ For broadside geometries, unusual effects are observed because the surface integral of perfect surfaces is identical to the linear integral over the edges.
} 


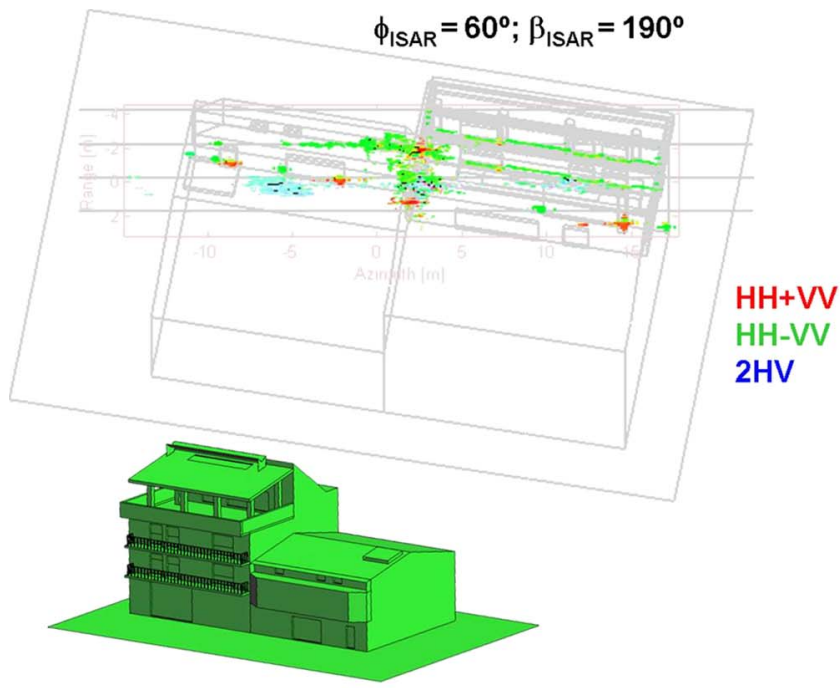

(a)

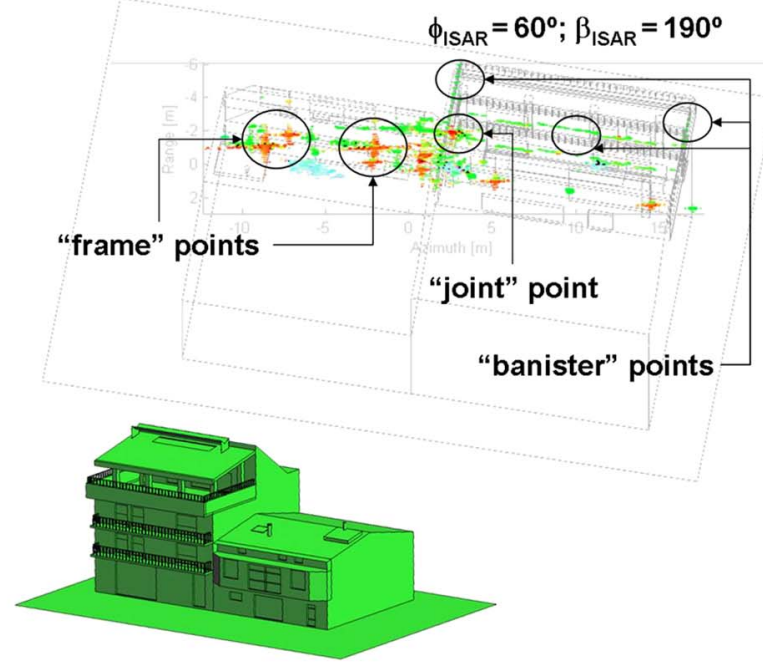

(b)

Fig. 5. RGB Pauli scattering maps obtained with $\phi_{\mathrm{ISAR}}=60^{\circ}$ and $\beta_{\mathrm{ISAR}}=190^{\circ}$ at C-band for two versions of building 1 model with (left) coarse and (right) fine details (see attached snapshots of models). The point of view is backward with near and far range at the top and bottom of the image. Dark lines help in a proper overlapping.

cause two undesired effects, namely: 1) strongly polarized mechanisms with extremely high RCS and 2) an incorrect simulation of dispersion, which is normally induced by 2-D corner scattering. To avoid these effects, one has to pay attention to corners and building's surfaces.

On the one hand, corners, either trihedral- or dihedralshaped, should not have perfect-square angles as the reflectivity response is too strong and has sidelobes that may interfere the surrounding targets. On the other hand, large perfectly flat planes at building surfaces (walls or floors) are extremely directives. An example is the 2-D wall-street corner interaction inducing a dihedral-like mechanism. If both surfaces are perfectly flat, the resulting RCS has a beamwidth that narrows with the size of the surface. This avoids a proper simulation of dispersion, as no surface roughness is considered.

To solve the previous problems, a type of surface roughness is simulated. This is done by adding a random offset to the coordinates of the points defining each model facet. In the current work, a normal distribution with zero mean and a standard deviation of $\sigma_{r}$ has been selected. As the purpose of surface roughness is to break with perfect geometries, the type of distribution has a negligible impact on the results.

To see the effect of surface roughness, Fig. 6 shows a comparison between the scattering maps (top) retrieved for two versions of building $2\left(\phi_{\mathrm{ISAR}}=60^{\circ}\right.$ and $\left.\beta_{\mathrm{ISAR}}=190^{\circ}\right)$, one with $\sigma_{r}=0 \mathrm{~cm}$ (perfect geometries), whereas the other with $\sigma_{r}=2 \mathrm{~cm}$ (added roughness). Snapshots of GRECO bitmap are also attached at the bottom in order to visualize the impact of surface roughness on EM calculations. There, white points highlight the pixels where multiple reflection takes place [11], [12]. In Fig. 6, the undesired effects due to perfect geometries are clear. The strong trihedral-like mechanisms dominating the scene in the first case migrate to a more realistic signature in the second one. Dispersive dihedral-like mechanisms due to ground-wall interactions are now sensed as well as the new trihedral-like mechanisms at the flat roof.
All the images presented in this paper consider surface roughness. $\sigma_{r}$ has been selected according to the density of small-scale details in each model so that no changes on the structure become noticeable. Tests show that acceptable values are $\sigma_{r}^{1}=0.5 \mathrm{~cm}$ for model 1 and $\sigma_{r}^{2}=2.0 \mathrm{~cm}$ for model 2 . For both models, the maximum facet length is fixed to $1 \mathrm{~cm}$ for achieving efficient roughness up to X-band [11].

\section{Material Information}

Fully dielectric materials cannot be simulated with the present version of the simulator, as GRECO can only process closed surfaces. This avoids the retrieval of the field transmitted through these materials, and, hence, the reflection of the incident field becomes a unique available operation. The complex dielectric permittivity defined for each material is included into the scenario via the Fresnel coefficients. This permits the simulation of the absorption and propagation loss. In this context, GRECO simulates the materials by adopting a thin lossy layer placed over the default perfect-conductor surface defining model geometry.

Obviously, material simulation needs further improvements. Other ways to include accurate dielectric information into the models could be evaluated, but they imply modifying the programming kernel of GRECO. This is something beyond the scope of this paper. As the main goal is to establish GS relations at $\mathrm{C}$ - and $\mathrm{X}$-band, the transmission field has a lower impact on the scattered fields, and, hence, the current material-simulation approach can be assumed to fulfill the work requirements. Fig. 1 shows the adopted materials.

\section{Time Dimension}

The variability of urban scenarios due to human activity may modify the measured polarimetric information inherent to the 


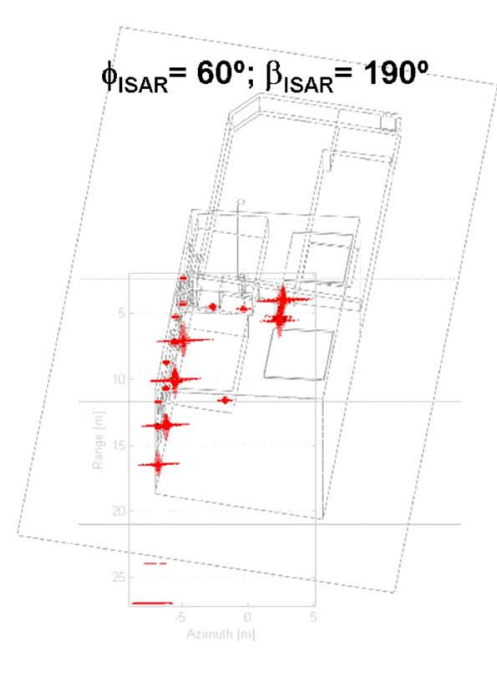

(a)

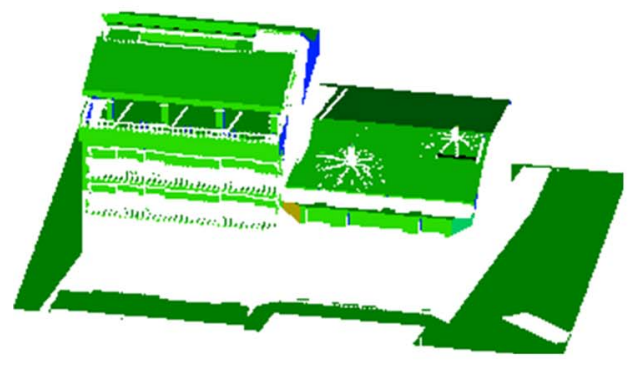

(c)

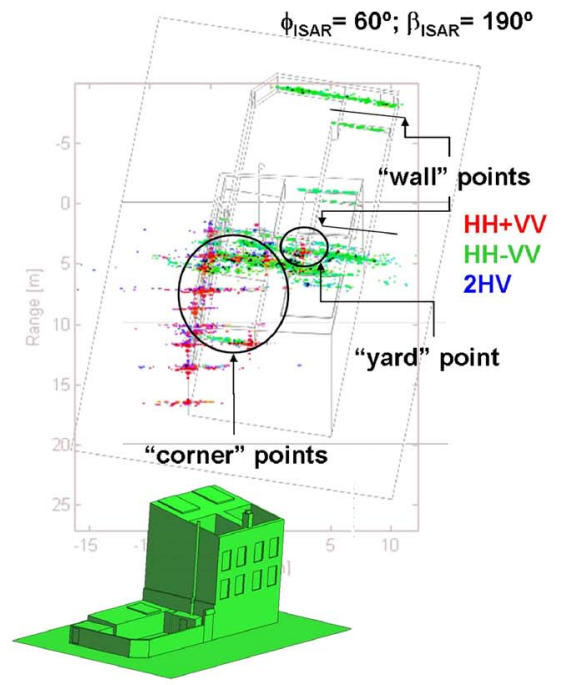

(b)

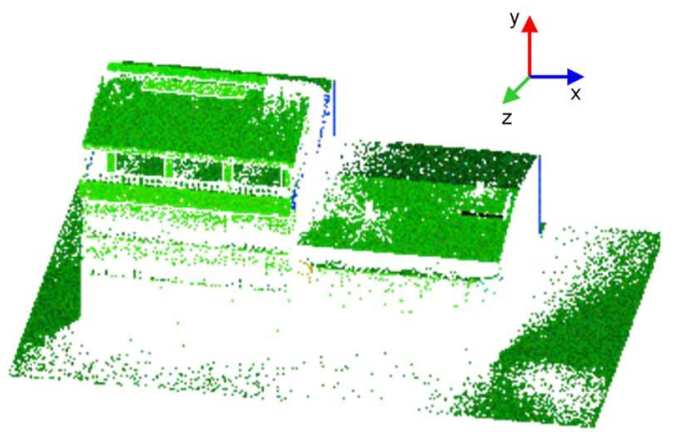

(d)

Fig. 6. RGB Pauli scattering maps obtained with $\phi_{\mathrm{ISAR}}=60^{\circ}$ and $\beta_{\mathrm{ISAR}}=190^{\circ}$ for two versions of building 2 model with (a) $\sigma_{r}=0 \mathrm{~cm}$ (perfect geometries) and with (b) $\sigma_{r}=2$ (surface roughness). They are complemented with (c), (d) the snapshots of GRECO bitmap for the same simulations processed with model 1. There, the coordinates of the normal vector of each visible facet is codified with an RGB-based color code. The reference system is centered at the lower right corner of the screen with " $\mathrm{z}$ " pointing to the user, " $y$ " to vertical, and " $\mathrm{x}$ " to horizontal. White pixels indicate the points with multireflection phenomena. The point of view is backward with near range at the top of the image. Dark lines help in a proper overlapping.

targets' geometry. Certainly, the scene can be geometrically modified (open/close doors, windows, blind, canopies, etc.) or can comprise new external elements which behavior is difficult to model (cars, cranes, scaffolding, etc.). With the improved resolutions of the new sensors, SAR images are more sensitive to such changes, and, consequently, a proper analysis within a simulation framework is essential to better model and understand the physics behind.

This topic was particularly important in the subsidence monitoring campaigns carried out with UPC's GB-SAR [9]. There, temporal stability was critical as the pixel-selection criteria was based on the persistence of the elemental Pauli mechanisms [8]. Therefore, a specific pixel was considered to have a high phase quality if it presented a stable polarimetric dispersion along the temporal interval. However, the analysis of real data sets have shown how human activity causes changes in the polarimetric behavior of pixels that were stable within a specific gap time. The pattern of the changes was diverse; some of them presented a periodicity, whereas others simply cause the mechanisms to disappear and reappear. This is going to be evaluated in the current work by processing versions of the two original models with slight structural changes.

\section{Simulated ScAtTERING MAPS}

This section analyzes scattering maps synthesized with the ISAR imaging geometry of Fig. 3 for the models in Fig. 1. $\beta_{\text {ISAR }}$ and $\phi_{\text {ISAR }}$ take different values according to typical imaging configurations that have a resolution of close to $15 \mathrm{~cm}$.

\section{A. Evaluation Along the Radar Aspect Angle}

Three main data sets have been used to analyze the influence of the aspect angle into urban scattering. They are, namely: 1) orientation set obtained at C-band for $\beta_{\mathrm{ISAR}}=\left\{170^{\circ}\right.$, $180^{\circ}, 190^{\circ}$, and $\left.200^{\circ}\right\}$ and $\phi_{\mathrm{ISAR}}=60^{\circ} ; 2$ ) incidence set obtained at C-band for $\beta_{\mathrm{ISAR}}=190^{\circ}$ and $\phi_{\mathrm{ISAR}}=\left\{40^{\circ}, 60^{\circ}\right.$, and $\left.75^{\circ}\right\}$; and 3) frequency set obtained at $\mathrm{C}$ - and X-band for $\beta_{\mathrm{ISAR}}=\left\{190^{\circ}\right.$ and $\left.200^{\circ}\right\}$ and $\phi_{\mathrm{ISAR}}=60^{\circ}$. Some samples processed with the Pauli theorem are provided in Figs. 7 and 8 with color-coded images (see Section III). ${ }^{4}$ The following points apply.

\footnotetext{
${ }^{4}$ The frequency set is not shown as the scattering maps at one operating band are almost identical with the others.
} 


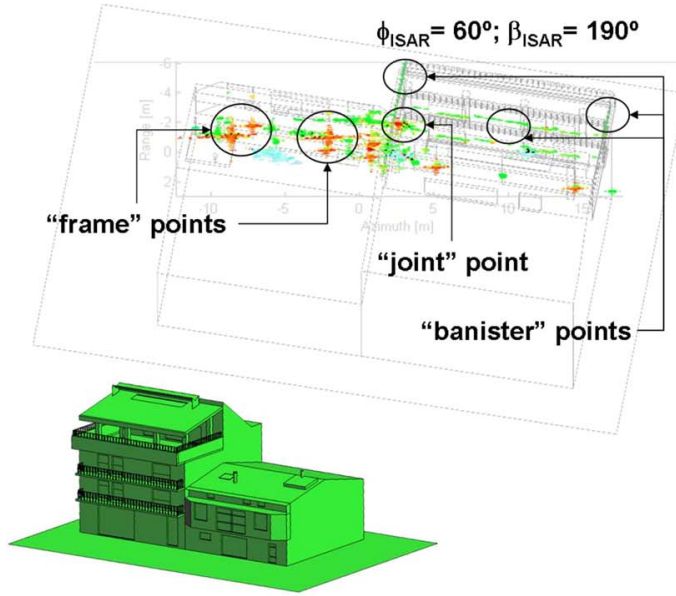

(a)

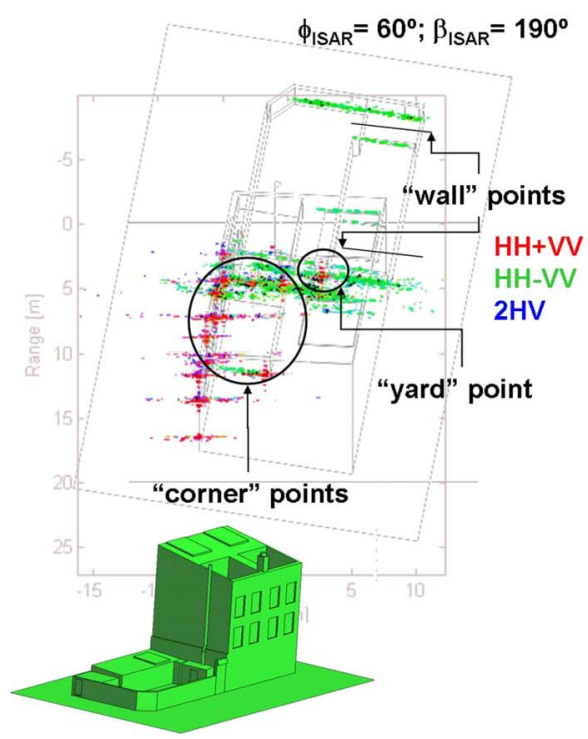

(c)

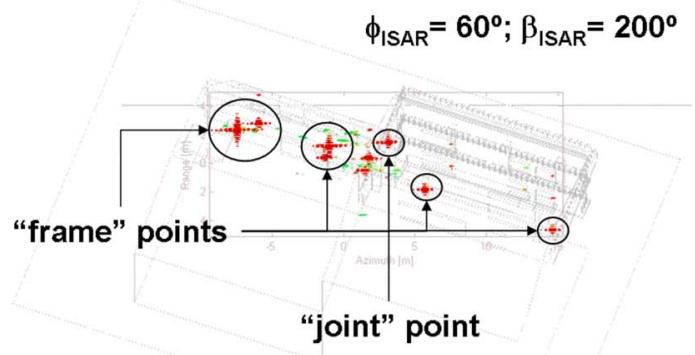

HH+VV HH-VV 2HV

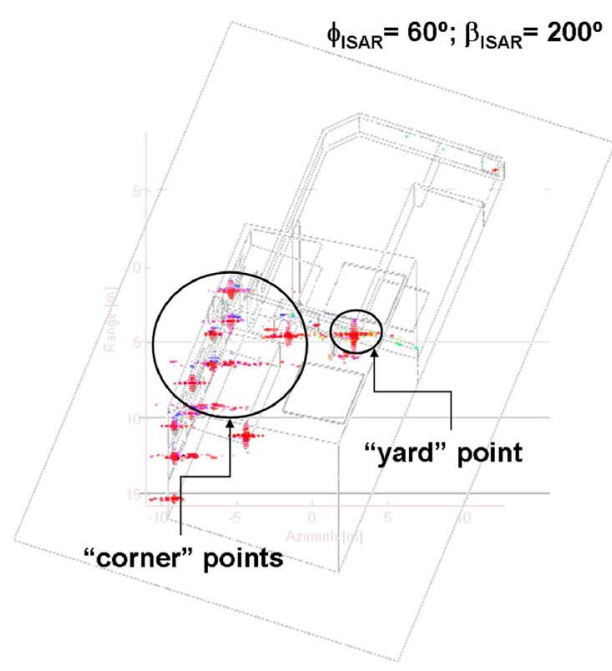

(d)

Fig. 7. RGB Pauli simulated scattering maps of the orientation set obtained at C-band and $\phi_{\text {ISAR }}=60^{\circ}$. Building models 1 and 2 are presented for (a), (c) $\beta_{\mathrm{ISAR}}=190^{\circ}$ and (b), (d) $200^{\circ}$. The point of view is backward with near range at the top of the image. Dark lines help on proper overlapping.

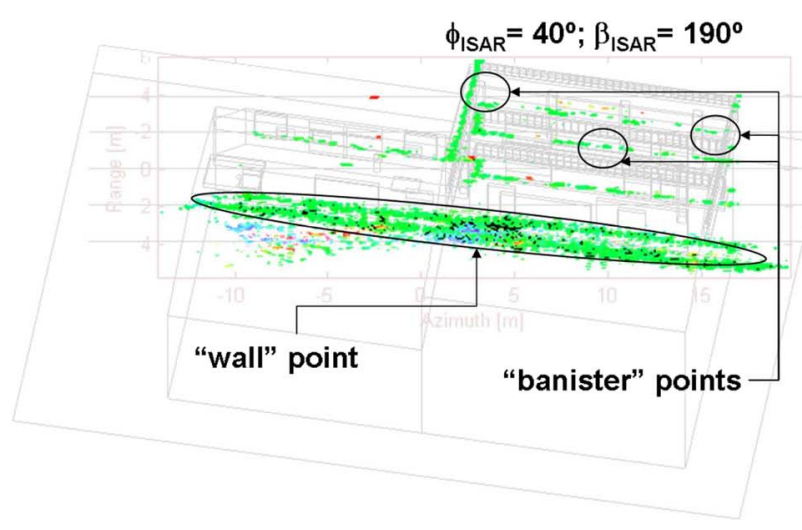

(a)

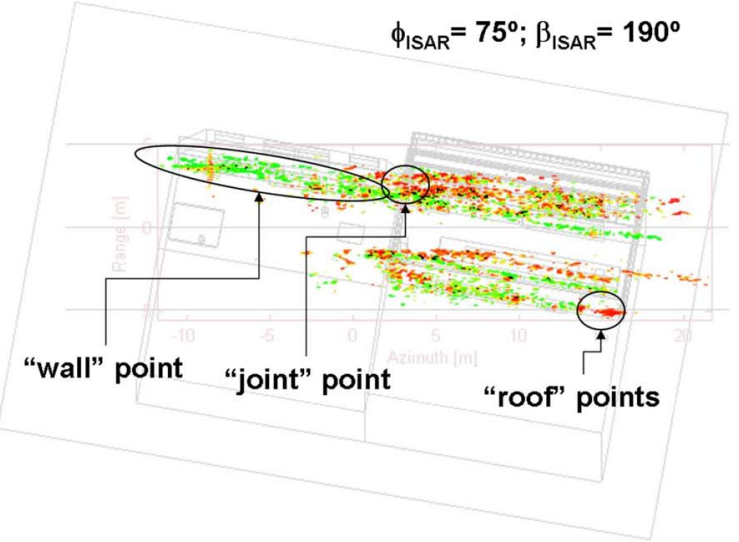

(b)

Fig. 8. RGB Pauli simulated scattering maps of the incidence set obtained at C-band and $\beta_{\text {ISAR }}=190^{\circ}$. Building model 1 is presented for (a) $\phi_{\mathrm{ISAR}}=40^{\circ}$ and (b) $75^{\circ}$. The point of view is backward with near-range at the top of the image. Dark lines help on proper overlapping.

1) Trihedral-like corners present high RCS and less sensitivity to the radar aspect angle. Some examples in model 1 are the corners located on window and door frames (frame point) and the $45^{\circ}$ beveled structure extruded from the facade of the smallest building (joint). In model 2, the scattering centers are on the yard (yard) and, again, 
on window frames (corner). All of these scatterers are tentative to be considered polarimetric persistent scatterers (PePS) in, for instance, DInSAR or buildingcharacterization applications. This is the extension of the concept outlined in [15] and [20] for ship scattering.

2) Dihedral-like corners are generated by wall-street and banister-floor interactions. In VHR systems, the wall-street mechanism is normally dispersive. However, it becomes more polarized (the mechanism is close to the canonical case) for incidences close to $40^{\circ}$ due to the alignment between the LOS and the maximum RCS axis. In contrast, the banister-floor mechanism presents a less dispersive character for a large range of incidences, i.e., it is, in general, more polarized. The reason may be on the reduced dimensions.

3) The polarimetric information seems to be more stable along the relative target orientation than along the incidence angle. In addition, the influence of multiscattering is not noticeable, and, hence, the response of each scatter is independent from the others. In the VHR context, this means that each resolution cell comprises the contribution of few scatterers, and, thus, the influence of time dimension could be less critical. This is an important conclusion not treated in the past and confirmed with the simulations presented here.

4) A certain polarimetric stability along the frequency is observed. Certainly, scatterer dimensions are large in terms of $\lambda$, and, hence, the results presented at C-band are similar to those at X-band. Note that this effect is not caused by the limitations of the simulator.

\section{B. Evaluation of the Time Dimension}

Time dimension has been evaluated by processing the original models with slight geometrical changes. In model 1, a garage door has been opened, whereas in model 2, a marquee with metallic posts has been added. ${ }^{5}$ These models have been evaluated at $\mathrm{C}$ - and $\mathrm{X}$-band for $\phi_{\mathrm{ISAR}}=60^{\circ}$ and $\beta_{\mathrm{ISAR}}=190^{\circ}$ and $200^{\circ}$. Some examples are shown in Fig. 9 with the color code fixed by the weight of the Pauli mechanisms. The results show that time dimension only affects the scattering response of the modified geometries as they backscatter independently from the others. Specifically, the scattering map of model 1 loses the trihedral-like scattering center at the base of the garage door as the associated trihedral-like corner disappears with the door opened. In contrast, the scattering map of model 2 has new dihedral-like mechanisms due to the posts.

Therefore, the effects of time dimension in SAR images depend on image resolution and scatterer distribution. For fine resolution, more localized changes are expected. This may allow new polarimetric selection criteria of persistent scatterers in DInSAR applications. In this context, advanced processing techniques, such as compressive sensing [21] or polynomial Fourier transform [22], could improve performance.

\section{Simulated SAR ImageS}

This section evaluates how the reflective information measured in the scattering maps is presented in SAR images. The scattering mechanisms are expected to be complexly combined according to the image resolution and scatterer distribution.

The previous sets (orientation, incidence, frequency, and temporal) have been processed at X-band for the imaging geometry of Fig. 4. The sensor is similar to TerraSAR-X with a resolution of $1.5 \mathrm{~m}$ in range and $2.5 \mathrm{~m}$ in azimuth. For the sake of simplicity, details regarding thermal noise and the equivalent sigma zero has been discarded. Fig. 10 shows some samples (for the same orientation) with the color code fixed by the weight of the Pauli mechanisms. The remaining cases provide similar results and have not been included. In general, it can be stated that the reflectivity information of simulated SAR images is similar to that of the scattering maps. More specifically, the following points apply.

1) The fine resolution of SAR images allows the processing of the reflectivity information in terms of target geometry and the isolation of some dispersion characteristics present in the scattering maps. Examples are the mechanisms related to frame and joint points in model 1 and those linked with trihedral-like corners and wall-street interactions in model 2.

2) Layover is an inherent phenomenon in urban areas, and its effects can be observed in both data sets. One example is the trihedral-like mechanism in the image of building 2 [see Fig. 10(b)]. This scatterer corresponds to a trihedrallike corner over the roof that appears focused close to the wall-street mechanism in near range.

The analysis of SAR images has shown that it is possible to find the PePS that have been previously characterized with the processing of scattering maps. The high precision in these data permits an accurate linking between specific scattering mechanisms and particular geometries. Quantitatively, these links can be formulated with parametric feature vectors, which may support new selection criteria. Therefore, when input SAR data is cross check with these reference feature vectors, certainty about the observed shape can be provided.

This methodology has been previously applied to ships. There, the marked target symmetry has made possible the development of a new classification approach [15], [20]. PePS selection relies on two conditions, namely: 1) PePS shall have the same dominant Pauli mechanism for a solid angle of at least $\pi / 3$ steradian and 2) the RCS associated with PePS shall be $10 \mathrm{~dB}$ higher than the surrounding scatterers. The application of this rule to ship-scattering maps has resulted in reference patterns for specific geometries. They have been parameterized with a feature vector $\Theta^{j}$ defined as [23]

$$
\Theta^{j}=\left\{\Theta_{n}^{j}\right\} \quad \text { for } 1<n<N
$$

where $N$ indicates the number of PePS in geometry $j$ and

${ }^{5}$ This marquee regards the bar terrace in front of building 2 (see Fig. 2).

$$
\Theta_{n}^{j}=\left\{x_{n}, y_{n}, z_{n}, p_{n}\right\}^{j}
$$




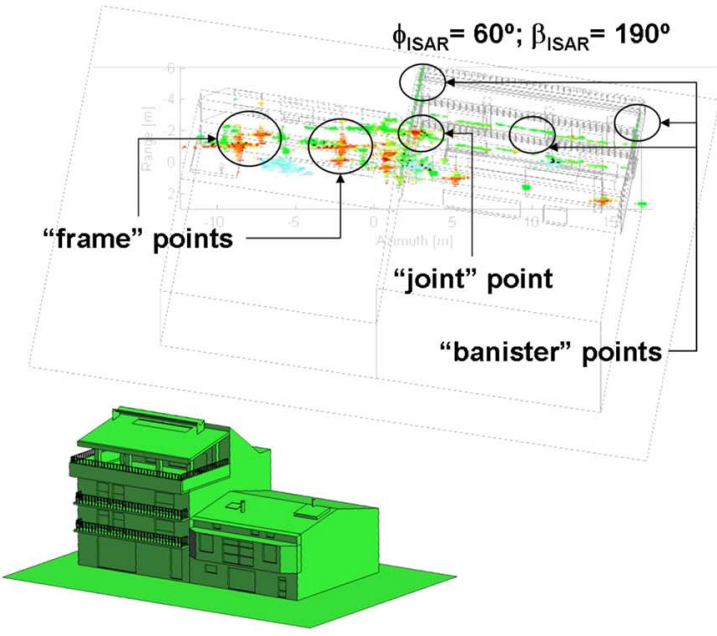

(a)

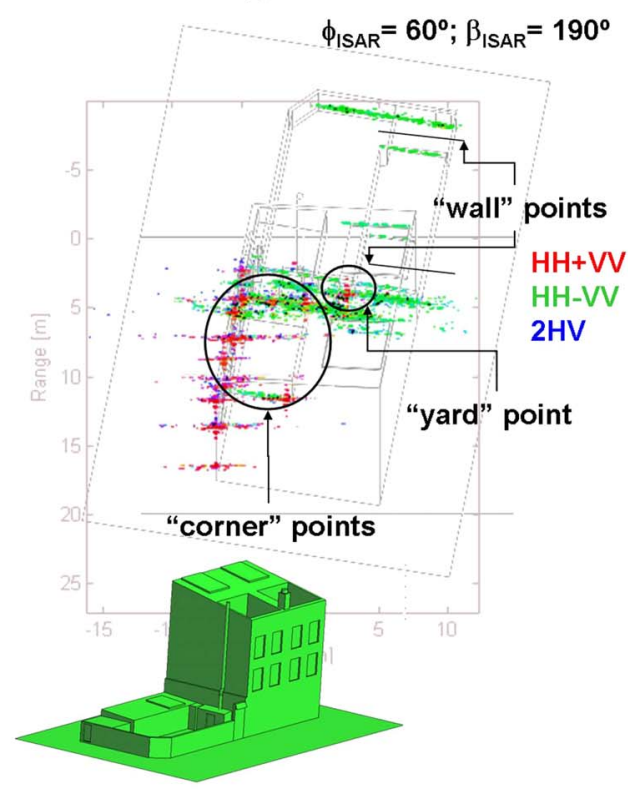

(c)

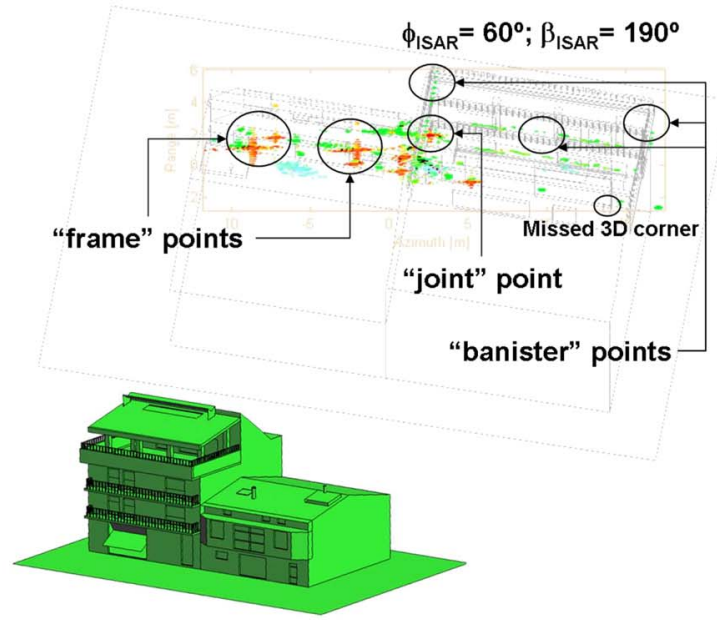

(b)

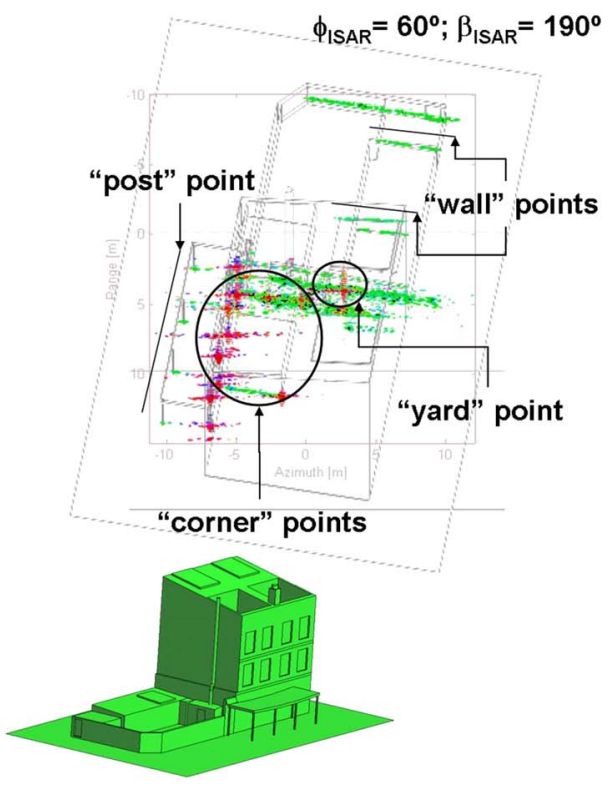

(d)

Fig. 9. RGB Pauli simulated scattering maps of the temporal set obtained at C-band for $\phi_{\text {ISAR }}=60^{\circ}$ and $\beta_{\text {ISAR }}=190^{\circ}$. Four models are processed. (a) Original building model 1. (b) Modified building model 1 with the garage door opened. (c) Original model 2. (d) Modified building model 2 with the added marquee (see the snapshots of models). The point of view is backward with near range at the top of the image. Dark lines help in a proper overlapping.

the PePS position expressed in a local system and the scattering mechanism $\left(p_{n}\right) . p_{n}$ is equal to zero, one, and two if the first, second, and third Pauli mechanisms are the dominant ones.

This formulation permits a quantitative framework for the simulation-based GS links between PePS and specific geometries. The values that the previous formulas can take will depend on the application in mind and the particular characteristics of the analyzed scatterers.

\section{REAL GB-SAR IMAGES}

This section uses GB-SAR data to validate the scattering information synthesized by the numerical tool GRECOSAR. The sets pertain to a subsidence monitoring campaign carried out by UPC in the neighborhood of Sallent (Fig. 2). Details about the GB-SAR sensor, processing scheme, images, and subsidence analysis can be consulted in [8] and [9].
In this section, the attention is focused on the image of Fig. 11, which shows a fully POLSAR set for an area with marked subsidence. The color code is fixed by the weight of the Pauli mechanisms but normalized to the span value. The image has been acquired at $\mathrm{X}$-band with a (range, azimuth) resolution of $(1.5,2) \mathrm{m}$, a linear synthetic aperture of $2 \mathrm{~m}$, and an incidence angle of $60^{\circ}$. The image is presented overlapped on a Google Earth's snapshot of the test area with a point of view similar to the GB-SAR imaging geometry. The optical image has been acquired before the measurement campaign, and slight differences can be detected with respect to the urban geometry measured by GB-SAR. Examples are the buildings demolished between optical and GB-SAR acquisitions that explain the lack of polarimetric information in some areas.

At a glance, it can be observed that the GB-SAR image presents a dispersion behavior very similar to what is measured by orbital sensors in urban areas. As in those cases, the main 


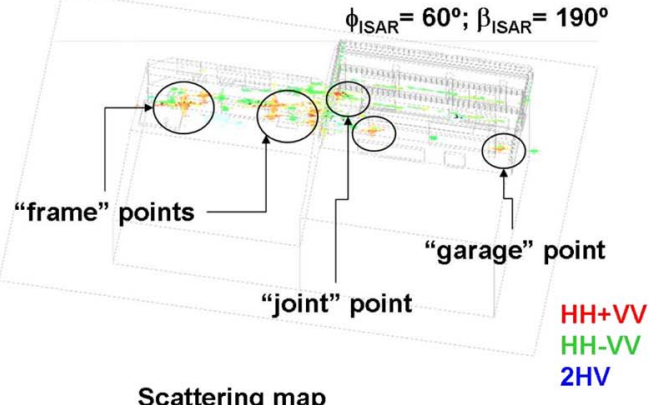

Scattering map

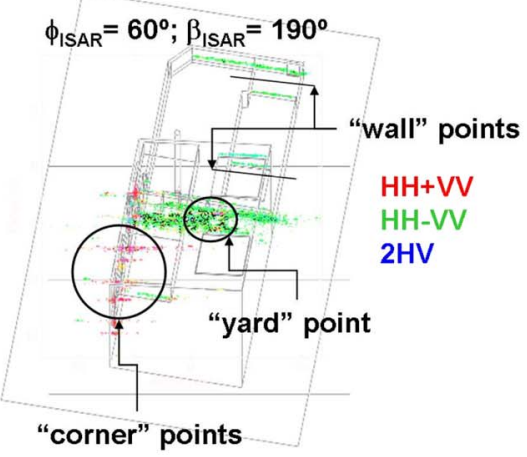

Scattering map

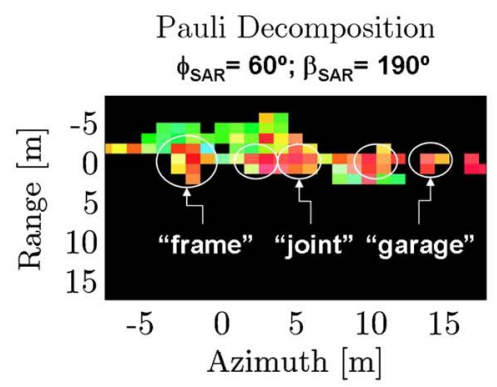

Simulated SAR image

(a)

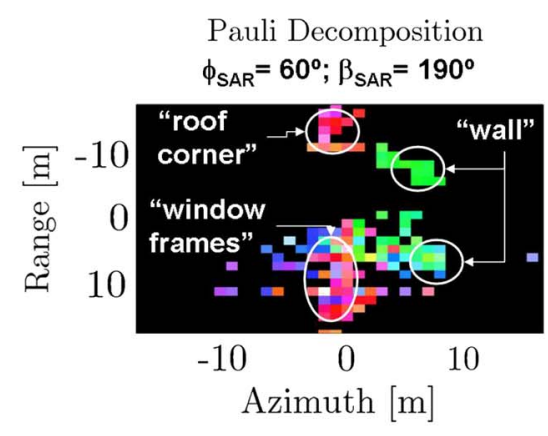

Simulated SAR image

(b)

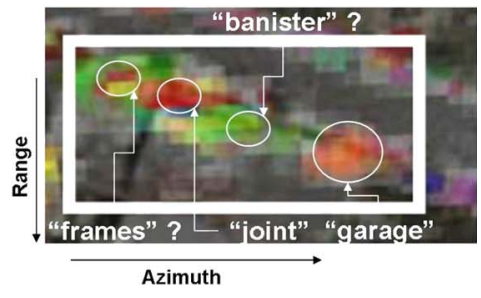

Real GB-SAR image

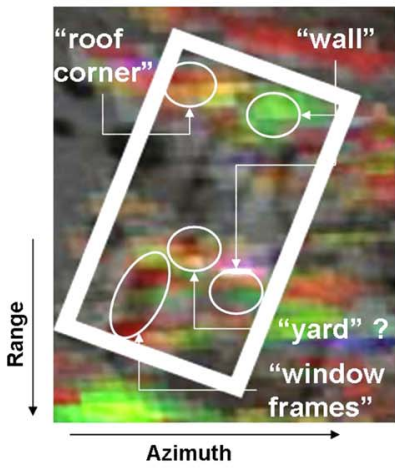

Real GB-SAR image

Fig. 10. RGB Pauli simulated SAR images obtained at X-band for $\phi_{\mathrm{SAR}}=60^{\circ}$ and $\beta_{\mathrm{SAR}}=190^{\circ}$. The associated simulated scattering maps and GB-SAR image have been attached for comparison. There, the response related to the modeled building have been isolated from the whole image of Fig. 11. The white rectangle delimitates approximately the image bounds of the simulated SAR image, whereas the white circles isolate some of the key spots in the scattering maps that allow linking the three types of images. All three images have the point of view backward with near range at the top.

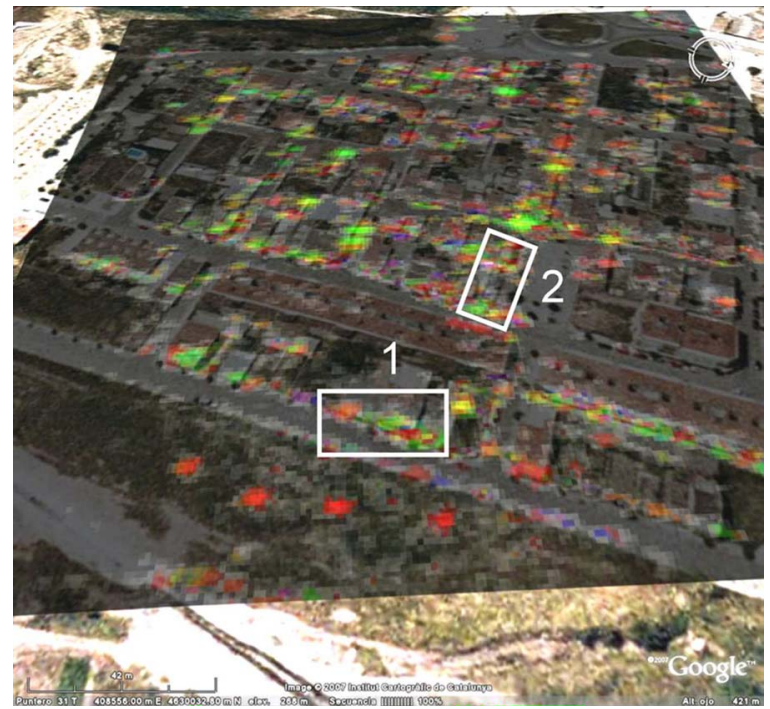

Fig. 11. Snapshot of a polarimetric reflectivity GB-SAR master image processed with the Pauli theorem and overlapped on a Google Earth snapshot of the test area. Color codification follows the convention of Figs. 5 and 6. White rectangles isolate the subimages shown in Fig. 10.

scattering centers are the dihedral-like mechanisms at the base of the building's facade and the trihedral-like mechanisms due to 3-D corners (mainly, window frames). The correlation of this image with the simulated ones (Fig. 10) points out also tight similarities. First, some key points can be identified in both data
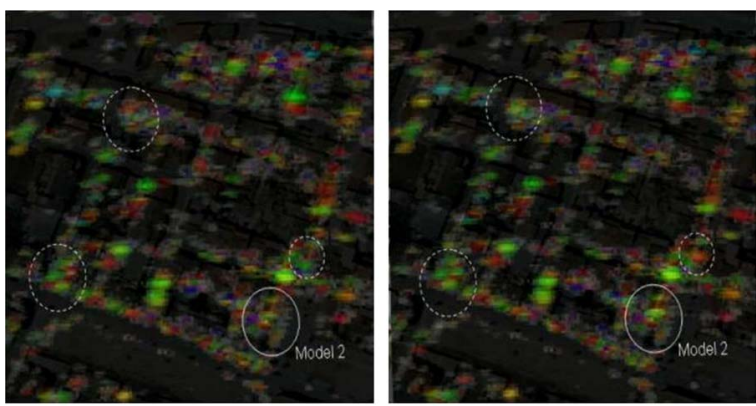

Time delay $20 \mathrm{~min}$

Fig. 12. Zoom of the image of Fig. 11 presenting the polarimetric-dispersion behavior measured for the same area at two acquisition times separated 20 min. As before, the image shows the weight of the Pauli mechanisms overlapped on the Google Earth snapshot with the same color code.

sets as the joint and frame scatterers in model 1 and the wall and window frame scatterers in model 2 (see the white circles). Their relative spatial distribution matches the one found in the simulated images with an accuracy lower than a resolution cell. The weight of their Pauli mechanisms have been also cross correlated, and the same numbers apply for both GB-SAR and simulated sets. The results show that the dominant mechanism of each key scatterer takes a normalized weight of around 0.7, whereas the remaining two were below 0.4 .

Regarding the effect of time dimension, Fig. 12 shows the zoom of an area measured by the GB-SAR sensor at two acquisition times. If compared with that presented in Section IV-B, 
the changes in the polarimetric signature follows the trend of what is obtained with GRECOSAR (sudden changes in the presence and reflectivity of scattering centers). Therefore, the way that time dimension has been simulated seems to be effective.

In summary, it appears that GRECOSAR performs well, and the synthesized urban images are close to real ones. The advantage is the high geometrical accuracy available with the scattering maps, which can be combined with real data to improve physical interpretation. Hence, instead of the mathematical manipulation of certain polarimetric descriptors, the analysis can be more focused to geometrical shapes, which are parametrized with feature vectors. This can be considered a more realistic approach.

\section{CONCLUSION}

This paper has presented a study that characterizes urban scattering from a geometrical point of view. Simulated and real data have been used to show that specific scattering centers can be accurately linked with particular geometrical shapes. Such GS links can be parameterized with a feature vector that permits the identification of these guide scatterers in real images. Image postprocessing and physical interpretation can be significantly improved with this information. This is particularly important for DInSAR subsidence studies where a new polarimetric framework may be feasible.

In a first step, fully polarimetric scattering maps and SAR images with very fine resolution have been synthesized with GRECOSAR for two models inspired in real buildings. The dispersion behavior has been analyzed in terms of the radaraspect angle, operating frequency, and time dimension, leading to the following conclusions.

1) The dominant mechanisms in urban scenarios seem to be the 2-D corners of wall-street interaction behaving as dihedrals and the 3-D corners at the terraces, balconies, or window frames, behaving as trihedrals.

2) The reflective behavior of buildings can be characterized by a finite and independent collection of elemental spots which distribution depends on building structure. The higher the resolution, the higher the sensitivity to time dimension, but the more localized the observed effects. Even most of the scatterers will preserve their properties with time; some of them can be modified due to changes on target's structure, like the opening and closing of a garage door or window.

3) Some spots (particularly 3-D corners) can be assumed to be polarimetric stable scatterers or PePS. Their application for urban-classification methods does not seem so straightforward than appears to be for ships. Instead, they seem to be more suited for DInSAR applications where geometrical-based feature vectors and polarimetric data can improve pixel selection and model adjustment. In the framework opened by the recently launched SAR sensors, this improvement is more important.

Simulator's data assessments have been performed with GB-SAR data. Quantitative comparisons with simulated sets have shown tight similarities in the polarimetric and spatial distribution of the main scattering centers. However, this initial work should be complemented with further studies that quantitatively evaluate the similarity between the feature vectors derived for specific scatterers from the reference scattering maps with those related to the same targets in GB-SAR data. For such purpose, a catalog of reference feature vectors should be defined for a set of well-known geometrical shapes via simulated scattering maps. The consistency of this reference list should then be validated with the analysis of real data with fine resolution and/or full polarimetric capabilities, such as those from TerraSAR-X or RADARSAT-2. The selection of specific areas of interest where ground-truth can be easily collected will make the overall process more straightforward.

Following this research line, first attempts on trying to include a selection criteria based on PePS in DInSAR studies have to be carried out. Therefore, the possible improvements that may be achieved could be reported and compared with the performance obtained with classical methods. The simulated data presented in this paper show that PePS should be initially located at the roofs and terraces.

\section{REFERENCES}

[1] V. Alberga, "Volume decorrelation effects in polarimetric SAR interferometry," IEEE Trans. Geosci. Remote Sens., vol. 42, no. 11, pp. 24672478, Nov. 2004.

[2] R. Z. Schneider, K. P. Papathanassiou, I. Hajnsek, and A. Moreira, "Polarimetric and interferometric characterization of coherent scatters in urban areas," IEEE Trans. Geosci. Remote Sens., vol. 44, no. 4, pp. 971-984, Apr. 2006.

[3] S. Guillaso, L. Ferro-Famil, A. Reigber, and E. Pottier, "Building characterization using L-band polarimetric interferometric SAR data," IEEE Geosci. Remote Sens. Lett., vol. 2, no. 3, pp. 347-351, Jul. 2005.

[4] S. R. Cloude and K. P. Papathanassiou, "Polarimetric SAR interferometry," IEEE Trans. Geosci. Remote Sens., vol. 36, no. 5, pp. 1551-1565, Sep. 1998.

[5] M. Jager, M. Neumann, S. Guillaso, and A. Reigber, "A selfinitializing POLInSAR classifier using interferometric phase differences," IEEE Trans. Geosci. Remote Sens., vol. 45, no. 11, pp. 3503-3518, Nov. 2007.

[6] D. Perissin and A. Ferretti, "Urban-target recognition by means of repeated spaceborne SAR images," IEEE Trans. Geosci. Remote Sens., vol. 45, no. 12, pp. 4043-4058, Dec. 2007.

[7] D. Perissin and F. Rocca, "High-accuracy urban DEM using permanent scatterers," IEEE Trans. Geosci. Remote Sens., vol. 44, no. 11, pp. 33383347, Nov. 2006.

[8] L. Pipia, X. Fabregas, A. Aguasca, and C. López-Martínez, "Atmospheric artifact compensation in ground-based DInSAR applications," IEEE Geosci. Remote Sens. Lett., vol. 5, no. 1, pp. 88-92, Jan. 2008.

[9] L. Pipia, X. Fabregas, A. Aguasca, C. López-Martínez, J. J. Mallorqui, and J. Marturia, "Polarimetric differential SAR interferometry: First results with ground-based measurements," IEEE Geosci. Remote Sens. Lett., vol. 6, no. 1, pp. 167-171, Jan. 2009.

[10] G. Margarit, J. J. Mallorqui, J. M. Rius, and J. Sanz-Marcos, "On the usage of GRECOSAR, an orbital polarimetric SAR simulator of complex targets, to vessel classification studies," IEEE Trans. Geosci. Remote Sens., vol. 44, no. 12, pp. 3517-3526, Dec. 2006.

[11] G. Margarit, "Marine applications of SAR polarimetry," Ph.D. dissertation, RSLab, Univ. Polytech. Catalunya, Barcelona, Spain, Jul., 2007.

[12] J. M. Rius, M. Ferrando, and L. Jofre, "High-frequency RCS of complex radar targets in real-time," IEEE Trans. Antennas Propag., vol. 41, no. 9, pp. 1308-1319, Sep. 1993.

[13] GID: International Center for Numerical Methods in Engineering (CIMNE). [Online]. Available: http://gid.cimne.upc.es/

[14] J. M. Rius, M. Ferrando, and L. Jofre, "GRECO: Graphical electromagnetic computing for RCS prediction in real time," IEEE Antennas Propag. Mag., vol. 35, no. 2, pp. 7-17, Apr. 1993.

[15] G. Margarit, J. J. Mallorqui, and X. Fabregas, "Single-pass polarimetric SAR interferometry for vessel classification," IEEE Trans. Geosci. Remote Sens., vol. 45, no. 11, pp. 3494-3502, Nov. 2007. 
[16] G. Margarit and J. J. Mallorqui, "Scattering-based model of the SAR signatures of complex targets for classification applications," Int. J. Navig. Observ., vol. 2008, pp. 426267-1-426267-11, Aug. 2008.

[17] D. R. Wehner, High Resolution Radar. Norwood, MA: Artech House, 1995.

[18] G. Margarit, J. J. Mallorqui, and C. Lopez-Martinez, "GRECOSAR, a SAR simulator for complex targets: Application to urban environments," in Proc. IEEE IGARSS, Jul. 2007, pp. 4160-4163.

[19] G. Margarit, J. Mallorqui, I. Corney, L. Pipia, C. López, and F. X. Fàbregas, "Analysis of urban areas scattering with simulated SAR imagery," in Proc. SP-649 ESA FRINGE Workshop, Nov. 2007, pp. 9-16.

[20] G. Margarit, J. J. Mallorqui, C. Lopez-Martinez, and J. Fortuny-Guasch, "Exploitation of ship scattering in polarimetric SAR for an improved classification under high clutter conditions," IEEE Trans. Geosci. Remote Sens., vol. 47, no. 4, pp. 1224-1235, Apr. 2009.

[21] E. Candes and M. Walkin, "An introduction to compressive sampling," IEEE Signal Process. Mag., vol. 25, no. 2, pp. 21-30, Mar. 2008.

[22] T. Thayaparan, I. Djurovic, and L. Stankovic, "Focusing distorted ISAR images using adaptive local polynomial Fourier transform," in Proc. IEEE IRS, 2006, pp. 1-4.

[23] G. Margarit, J. J. Mallorqui, C. Lopez-Martinez, and J. Fortuny-Guasch, "Phenomenological vessel scattering study based on simulated inverse SAR imagery," IEEE Trans. Geosci. Remote Sens., vol. 47, no. 4, pp. 1212-1223, Apr. 2009.

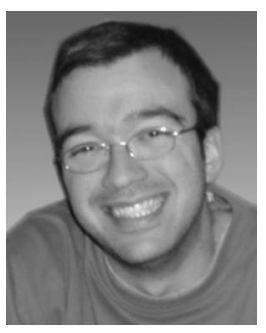

Gerard Margarit (S'06-M'07) was born in Barcelona, Spain, in 1979. He received the B.S. degree in electrical engineering, and the Advanced Studies, MERIT M.S., and Ph.D. degrees with European Mention from the Universitat Politècnica de Catalunya (UPC), Barcelona, in 2002, 2004, 2007, and 2007, respectively.

From 2003 to 2006, he had an official grant from the Science and Education Spanish Department (FPU), and became an Assistant UPC Researcher in 2007. In 2004, he made a four-month stage with the Joint Research Centre working on oil spill monitoring. Since 2008, he has been with the Science and Earth Observation Processing Systems, GMV Aerospace and Defense, Barcelona. He has been involved in different projects addressing ship-detection and classification, critical infrastructure monitoring, forest mapping, waste management, and precision agriculture. His current research interests are in the fields of radar image-processing techniques, SAR simulation in multiple scenarios, wind and ocean-current mapping, and SAR applications in forestry and agriculture.

Dr. Margarit was the recipient of the extraordinary UPC Award for the best $\mathrm{Ph} . \mathrm{D}$. in the field of Communication and Information technologies.

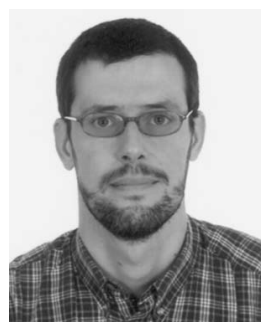

Jordi J. Mallorquí (S'93-M'96) was born in Tarragona, Spain, in 1966. He received the Ingeniero degree and the Doctor Ingeniero degree in telecommunications engineering for his research on microwave tomography for biomedical applications from the Universitat Politècnica de Catalunya (UPC), Barcelona, Spain, in 1990 and 1995, respectively. He was with the Department of Signal Theory and Communications as a Ph.D. student, in 1991.

In 1993, he was an Assistant Professor, and since 1997, he has been an Associate Professor with the Telecommunications Engineering School. His teaching activity involves microwaves, radionavigation systems, and remote sensing. He spent a sabbatical year with the Jet Propulsion Laboratory, Pasadena, CA, in 1999, working on interferometric airborne SAR calibration algorithms. He is currently working on the application of SAR interferometry to terrain-deformation monitoring with orbital, airborne, and ground data, vessel detection and classification from SAR images, and 3-D electromagnetic simulation of SAR systems. He is also collaborating in the design and construction of a ground-based SAR interferometer for landslide control. Finally, he is currently developing the hardware and software of a bistatic opportunistic SAR for interferometric applications using ERS, ENVISAT, and TerraSAR-X as sensors of opportunity. He has published more than 80 papers on microwave tomography, electromagnetic numerical simulation, and SAR processing, interferometry, and differential interferometry in referred journals and international symposia.

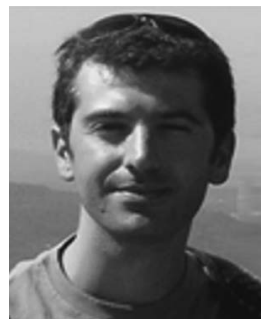

Luca Pipia (S'02-M'09) was born in Sassari, Italy, in July 1976. He received the B.S. degree (cum laude) in electrical engineering from the Università degli Studi di Cagliari, Cagliari, Italy, in 2002, and the Ph.D. degree in polarimetric differential SAR interferometry (PolDIInSAR) from the Universitat Politècnica de Catalunya (UPC), Barcelona, Spain, in 2009.

He was with the High-Frequency Institute, German Aerospace Center (DLR), Oberpfaffenhofen, Germany, from June 2001 to December 2001, where he worked on land classification using polarimetric SAR (POLSAR) information. From 2003 to 2009, he was with the Remote Sensing Laboratory, Departament de Teoria del Senyal i Comunicacions, UPC, where he was deeply involved in the development and assessment of a novel polarimetric formulation of coherence-based advanced differential SAR interferometry (DinSAR) techniques using real ground-based POLSAR data. He is currently with the Remote Sensing group, Institut Cartogràfic de Catalunya (ICC), Barcelona. His research interests deal with SAR polarimetry, DinSAR, and, more recently, with the retrieval of quantitative information from hyperspectral thermal-infrared airborne data.

Dr. Pipia has served as a reviewer for the IEEE GEOSCIENCE AND REMOTE SENSING LETTERS. 